\title{
Severe mortality in mesocosm-reared sharpsnout sea bream Diplodus puntazzo larvae due to epitheliocystis infection
}

\author{
Pantelis Katharios*, Maria Papadaki, Nikos Papandroulakis, Pascal Divanach \\ Institute of Aquaculture, Hellenic Centre for Marine Research, PO Box 2214, Heraklion 71003, Crete, Greece
}

\begin{abstract}
This paper describes severe mortalities recorded in sharpsnout sea bream Diplodus puntazzo larvae reared in mesocosms. The mortalities were attributed to epitheliocystis infection. The pathology associated with the disease is described using histological techniques. Microscopical examination showed a massive infection of the skin, fins, and oral cavity, with impaired feeding, respiration, and osmoregulation being the most likely cause of death. This is the first report of epitheliocystis disease in sharpsnout sea bream and in fish at such an early developmental stage.
\end{abstract}

KEY WORDS: Epitheliocystis · Chlamydia $\cdot$ Sea bream $\cdot$ Fish larva $\cdot$ Disease $\cdot$ Mortality Resale or republication not permitted without written consent of the publisher

\section{INTRODUCTION}

Epitheliocystis is an infectious disease affecting the gills and the integument of many marine and freshwater fish species (Nowak \& LaPatra 2006). It is caused by Gram-negative bacteria, recently characterized as Chlamydia (Order: Chlamydiales), forming intracellular inclusions (Draghi et al. 2007). These inclusions progressively enlarge, resulting in hypertrophied cells that resemble spherical cysts. Although the pathogen cannot be cultured, and therefore Koch's postulate with experimental challenges has yet to be demonstrated, mortalities have been associated with the presence of the bacterium (Paperna 1977, Bradley et al. 1988, Venizelos \& Benetti 1996, Crespo et al. 2001, Goodwin et al. 2005). Mortalities (4 to $100 \%$ ) and prevalence (20 to $100 \%$ ) of the disease varies depending on fish species, developmental stage of the host, and intensity of infection. Mortality has been reported only in cultured fish, with early life stages being more susceptible (Nowak \& LaPatra 2006). The pathology associated with epitheliocystis includes epithelial hyperplasia, inflammation of the affected tissue, proliferation of the mucous cells, and necrosis; however, in many cases, the cysts provoke no cellular response (Paperna 1977, Grau \& Crespo 1991, Crespo et al. 1999, 2001, Nowak \& Clark 1999).
Sharpsnout sea bream Diplodus puntazzo (Cetti, 1777) has been successfully introduced to Mediterranean aquaculture, and it is a species with high market value (Divanach et al. 1993, Sara et al. 1999, Papandroulakis et al. 2004). It is a species with high growth rates that, while easily reared in early life stages, has high mortality rates in on-growing facilities mainly due to diseases (Athanassopoulou et al. 1999, Munoz et al. 2007). In this paper we describe a case of severe mortalities of sharpsnout sea bream larvae reared in the semi-intensive hatchery of the Institute of Aquaculture, HCMR, Crete, attributed to epitheliocystis hyperinfection. This is the first report of epitheliocystis in this species and the first report of the disease in such early fish larvae (pre-flexion stage).

\section{MATERIALS AND METHODS}

Rearing. Sharpsnout sea bream were reared in the facilities of the Institute of Aquaculture using semiintensive mesocosm technology (Papandroulakis et al. 2004). Five $40 \mathrm{~m}^{3}$ tanks were stocked consecutively with sharpsnout sea bream eggs at a density of approximately $8 \mathrm{l}^{-1}$. The tanks were filled with natural sea water (salinity $40 \mathrm{ppt}$ ), which was renewed from a lit- 
toral well (salinity $32 \mathrm{ppt}$ ) at a rate that progressively increased from $5 \%$ daily at the beginning to $50 \%$ daily by $25 \mathrm{~d}$ post hatching (dph). The fish were fed on enriched rotifers Brachionus plicatilis and enriched Artemia sp. nauplii, while microalgae Chlorella minutissima were added daily in the rearing water. Temperature ranged between 21 and $23^{\circ} \mathrm{C}$.

Epizootiology of infection. The earliest mortalities among sharpsnout sea bream larvae were recorded at $7 \mathrm{dph}$ while the latest (in terms of mortality onset) were recorded at $20 \mathrm{dph}$. All 5 tanks were severely affected by the disease with final mortalities reaching $100 \%$ within $25 \mathrm{~d}$. Moribund fish were lethargic and cachectic, swimming at the surface of the water. Sampling to assess the intensity and prevalence of infection was performed every second day, and 15 to 20 fish from each population were examined fresh under a stereoscope.

Histology. Twenty larvae (15 dph) with signs of the disease (spherical cysts in the integument) were killed with a high dose of the anesthetic 2-phenoxyethanol and preserved for histology.

For the histological examination, the larvae were fixed in 4:1 formaldehyde:glutaraldehyde (McDowell \& Trump 1976), dehydrated in a 70 to $95 \%$ ethanol series, and embedded in glycol methacrylate resin (Technovit 7100, Heraeus Kulzer). Serial sections were obtained at a thickness of 3 to $5 \mu \mathrm{m}$ on a microtome (Leica RM 2245) using disposable blades. After drying, slides were stained with methylene blue/azure II/basic fuchsin (Bennett et al. 1976), Gram stain, and Giemsa and examined under a light microscope (Leica DMLB). Microphotographs were taken with an ALTRA 20 digital camera attached to the microscope and analyzed using the ImagePro 6.1 (MediaCybernetics) image analysis system.

\section{RESULTS}

All fresh specimens grossly examined under the stereoscope had numerous cysts in the skin and the oral cavity (100\% prevalence), with the majority of the fish being hyper-infected. Most of the live larvae that were grossly examined had more than 30 cysts in the integument. The cysts were circular and ellipsoid with yellowish and brownish coloration (Fig. 1).

Light microscopy observations under high magnification $(\times 1000)$ of both fresh and sectioned specimens revealed that the cysts were actually intracellular granular inclusions containing particles resembling the elementary and reticulate bodies described in chlamydial infections and specifically epitheliocystis (Fig. 2). The cysts were stained Gram-negative and Giemsa-positive (Fig. 3).

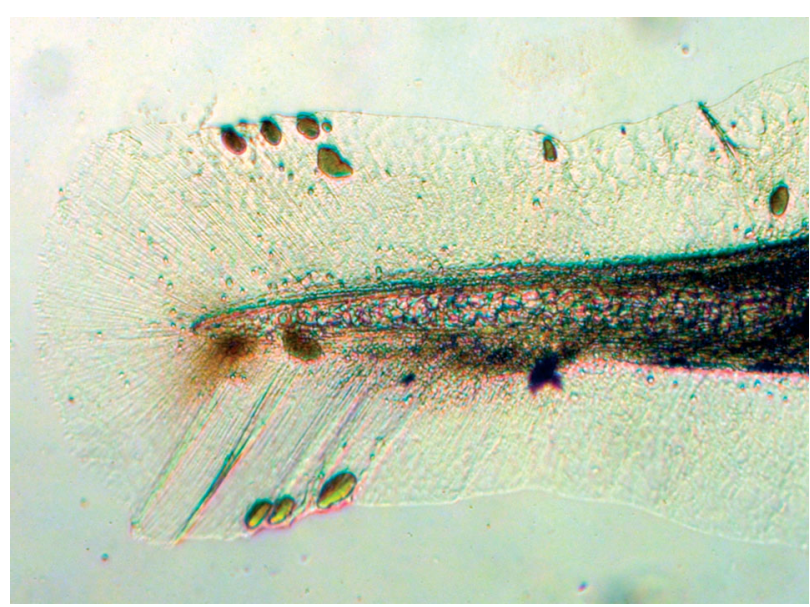

Fig. 1. Diplodus puntazzo. Caudal primordial fin of a larva at the flexion stage with numerous yellow and brown ellipsoid cysts
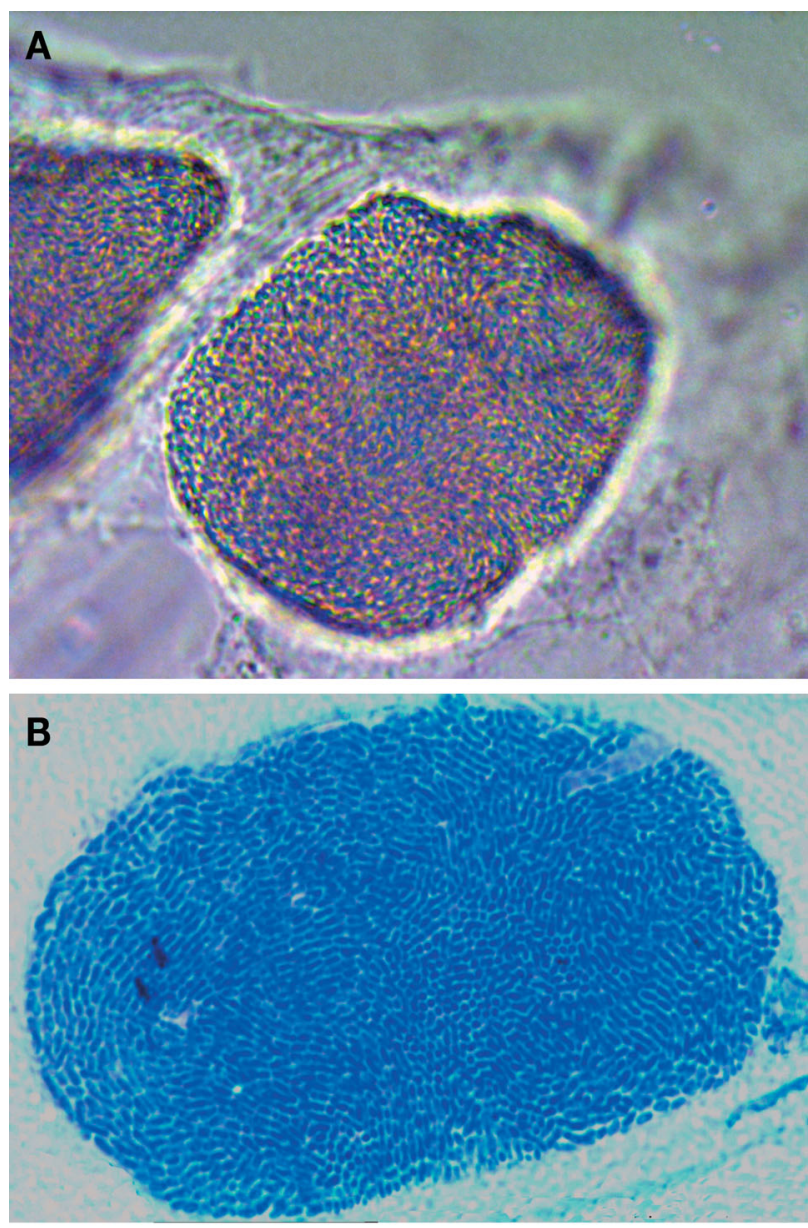

Fig. 2. Diplodus puntazzo. High power magnification $(\times 1000)$ micro-photographs of epitheliocystis inclusions from larvae. (A) Fresh mount of the inclusion, (B) cross section of tissue containing the inclusion. Note the various shaped particles within the cyst resembling the elementary and reticulate bodies characteristic of the Chlamydia developmental cycle 


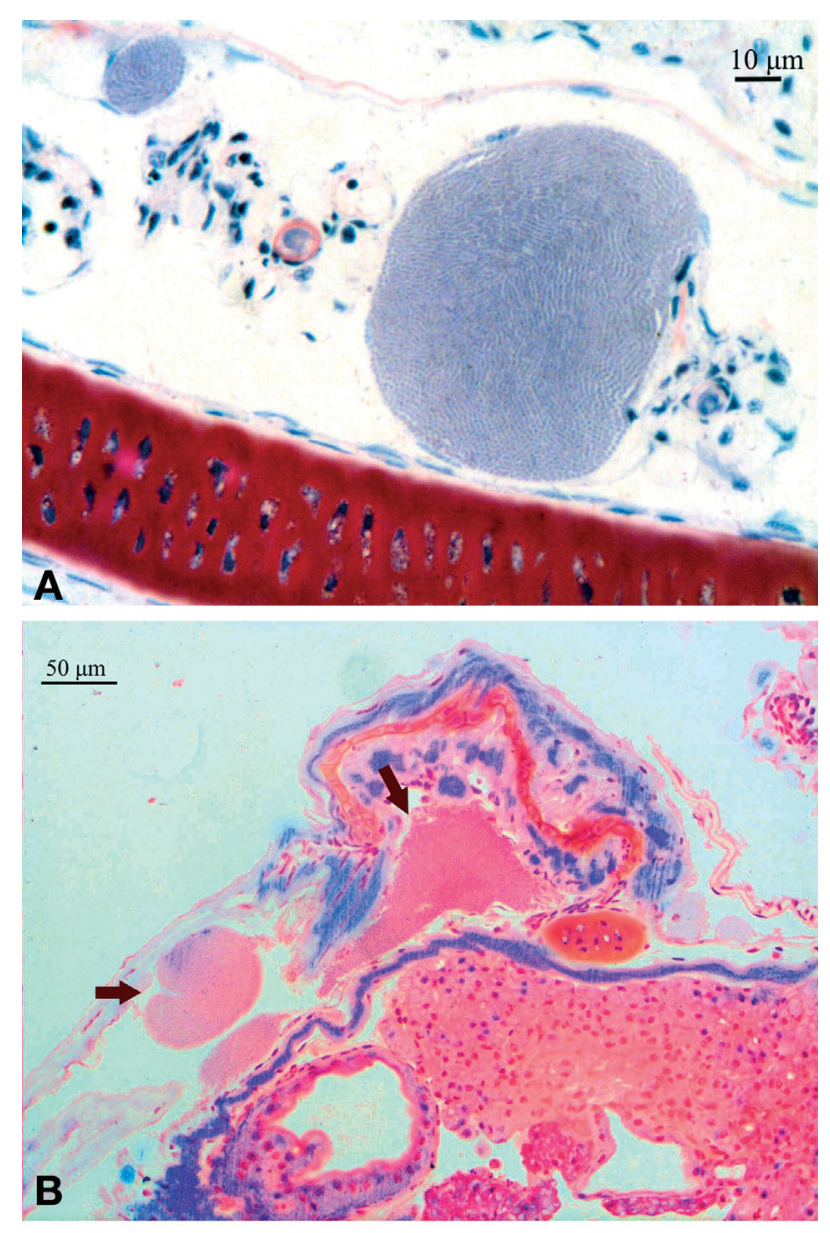

Fig. 3. Diplodus puntazzo. (A) Cross section of the head area of a larva with 2 inclusions positively stained with Giemsa.

(B) Gram-negative stained epitheliocystis (arrows)

The ellipsoid epitheliocystis inclusions varied in size ranging between $16 \times 21 \mu \mathrm{m}$ (minor $\times$ major axis) and $89 \times 115 \mu \mathrm{m}(\mathrm{n}=25)$. The inclusions were located mainly on the primordial fins, in the skin epithelium, and in the oral cavity (Fig. 4). All cysts identified in the histological sections had a granular texture and were never observed to provoke any cellular response of the host such as hyperplasia or increased cellularity. The only host response recorded was the encapsulation of the cyst within a thin layer of squamous epithelial cells. In heavily infected fish, the encapsulated cysts embedded in the epithelium of the oral cavity physically blocked the entrance of the mouth (Fig. 5) and the esophagus (Fig. 4B).

\section{DISCUSSION}

To our knowledge, this is the first report of epitheliocystis disease in sharpsnout sea bream Diplodus pun-

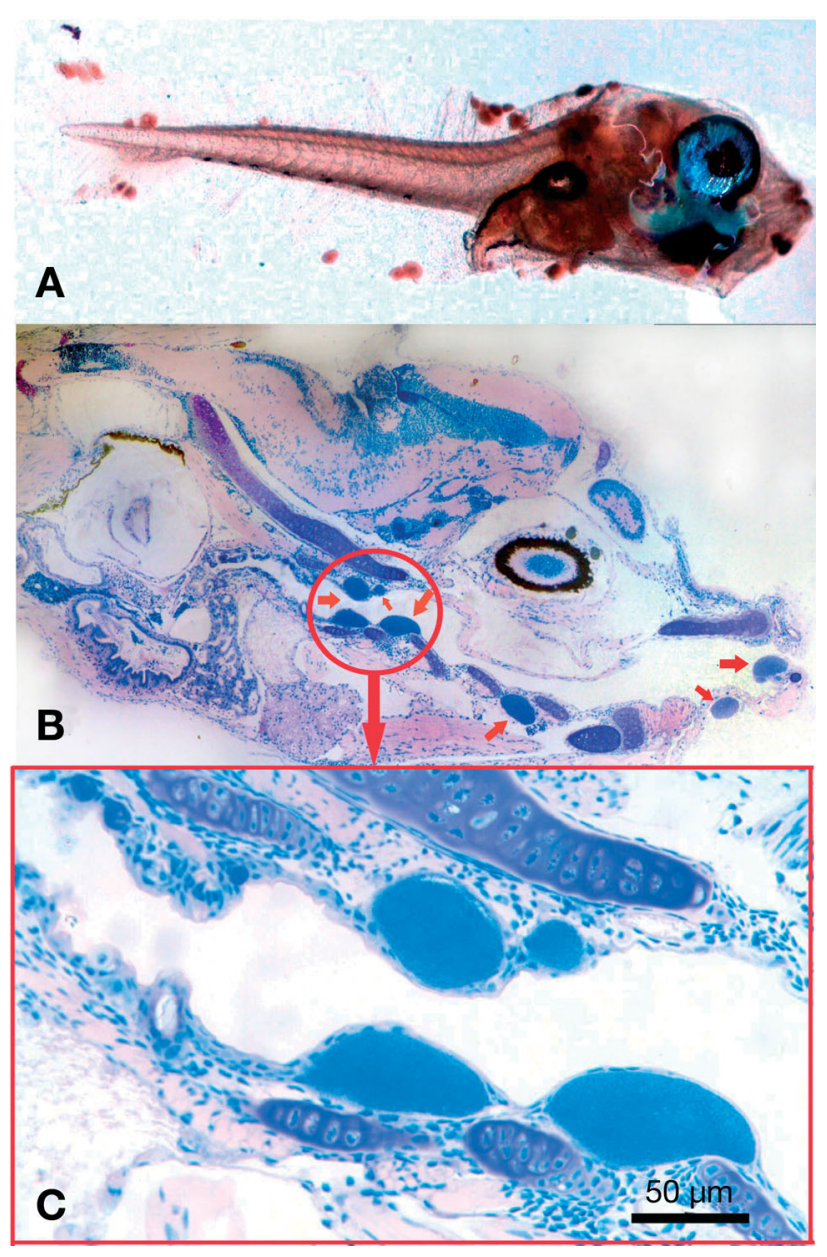

Fig. 4. Diplodus puntazzo. (A) Larva bearing numerous cysts in the primordial fins. (B) Cross section of an infected larva showing cysts within the oral cavity (arrows). (C) Detail of the cysts within the oral cavity rostral to the esophagus. Note that the cysts are surrounded by a limiting membrane and/or a thin layer of squamous epithelial cells without eliciting any significant host response

tazzo and the first report of the disease affecting fish at such an early developmental stage (pre-flexion/flexion). The earliest reported stage of fish affected by epitheliocystis infection was the metamorphosis stage (28 to $42 \mathrm{dph}$ ) of the Pacific yellowtail Seriola mazatlana (Venizelos \& Benetti 1996). The prevalence of epitheliocystis has been positively correlated with age in wild winter flounder Pleuronectes americanus (MacLean 1993), a supposition questioned by Nowak \& Clark (1999), who in contrast observed a negative correlation of epitheliocystis prevalence and age in Atlantic salmon Salmo salar. Instead, those authors proposed temperature as the main factor affecting the prevalence of epitheliocystis, with higher temperatures enhancing the progress and the development of the disease. On the other hand, Crespo et al. (1990) 


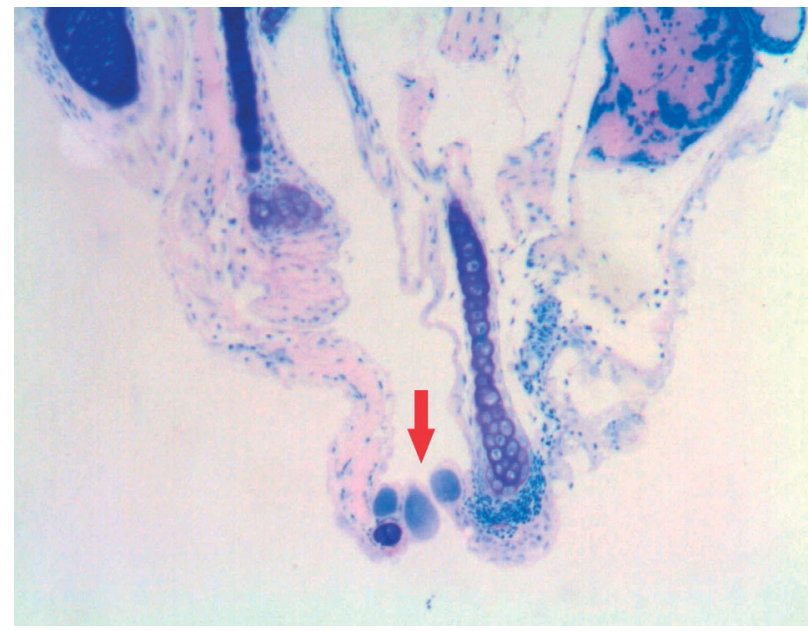

Fig. 5. Diplodus puntazzo. Cross section of a larval head area showing 3 inclusions (arrow) mechanically blocking the opening of the oral cavity

related the high mortalities due to epitheliocystis in greater amberjack Seriola dumerili to low temperatures, with the caveat that the fish were heavily parasitized by trichodins and blood flukes, which could also have contributed to high mortalities. Our observations are in agreement with Nowak \& Clark (1999), since a massive infection of epitheliocystis was observed in early life stages of sharpsnout sea bream. Furthermore, the onset of the disease was recorded in September when the water temperature in Crete is very high (21 to $23^{\circ} \mathrm{C}$ ) and epitheliocystis was the only pathological finding.

Aquaculture conditions have been claimed to favor the prevalence of the disease. Several authors have indicated that farmed fish are much more susceptible to epitheliocystis than wild fish (Paperna 1977, Grau \& Crespo 1991, Nowak \& LaPatra 2006). This is probably due to increased fish densities, presence of nutrients, and stress. In our case, the fish were reared using a mesocosm protocol, which uses low fish densities, but requires increased nutrient concentrations in the water. This suggests that low fish densities alone cannot protect the fish from the disease in aquaculture, and nutrient concentration may well be a critical risk factor.

Controversial reports on the effect of epitheliocystis in the host have been published. Several authors have described significant proliferative reactions, with severe epithelial cell hyperplasia being the most common observation focused mainly in the lamellae of the gills and pseudobranch (Paperna 1977, Bradley et al. 1988, Goodwin et al. 2005). In other cases, the host response is benign, expressed as limited hyperplasia and encapsulation of the cyst with one or more concentric squamous epithelial layers (Turnbull 1993). Host response to epitheliocystis is related to the developmental stage of the pathogen, which according to Crespo et al. (1999) can be differentiated by the texture of the cyst. Granular inclusions that are assumed to contain the immature stages of the pathogenic agent are usually found in younger fish and cause little or no host response. On the other hand, amorphous inclusions contain mature stages of the pathogen and in most cases provoke hyperplastic cellular response. These inclusions are mostly found in older fish. In our case, sharpsnout sea bream larvae exhibited only granular cysts that provoked no host response apart from the encapsulation of the cysts within a thin layer of epithelial cells. Despite the fact that no electron microscopy was performed in our samples, it is interesting that the cysts observed under high magnification $(\times 1000)$ showed a particle pattern that is more similar to the elongated and small cells described by Crespo et al. (1999) in the amorphous inclusions observed with transmission electron microscopy (Fig. 2). This could be explained by the diversity of the morphology of epitheliocystis in different host species as indicated in a review by Nowak \& LaPatra (2006).

Mortalities due to epitheliocystis infection have been associated mainly with the proliferative host response in older fish resulting in hyperplasia of the gill lamellar epithelium, distortion of the tissue structure, inflammation, necrosis, and therefore impairment of the gas transfer and osmoregulation processes (Crespo et al. 1990, Nowak \& LaPatra 2006). In younger fish, although the predominant form of the cyst is granular, which, as mentioned earlier, provokes little or no host response, the mortalities, surprisingly, are much higher. In early developmental stages, the morphological and functional changes influence organism performance. Specifically, at the larval stage, fish undergo ontogenetic changes necessary for establishing exogenous feeding such as the opening of the mouth, the completion of the functionality of the oral cavity, the onset of digestive enzyme production, and the alimentary canal formation. Sharpsnout sea bream develop a perfectly functioning mouth by 6 dph (Faranda et al. 1985). Moreover, during the larval stage, sharpsnout sea bream are equipped with free neuromast and olfactory ciliated receptor cells, making the rostral area of the head the most sensitive region of the body (Boglione et al. 2003). In our case, the most affected areas of the examined fish were the skin, the fins, and the head area (outer and buccal and gill cavity). Many fish had numerous cysts in the mouth opening and alongside the oral cavity up to the opening of the esophagus. This could affect fish either mechanically by physically blocking the entrance of the food items leading to death due to reduced food intake at a very sensitive developmental stage or by obstructing the 
development or the function of sensory cells that are implicated in larval food detection (Boglione et al. 2003).

Furthermore, in the early developmental stages of teleosts, the gills are not fully developed; instead, the skin, apart from its protective role, has vital physiological functions such as osmoregulation, respiration, and excretion and therefore is much more important (Varsamos et al. 2005). It should be noted that fully developed gills were not observed in any of the sharpsnout sea bream larvae examined by histology. However, in many cases, epitheliocystis inclusions were found near the area where gill filaments would develop (data not shown). On the other hand, skin epithelium was the tissue affected most. It can be therefore logically assumed that mortalities in epitheliocystis-infected sharpsnout sea bream could be also attributed to damage and/or coverage of the skin by the cysts. In the first report of epitheliocystis in the literature (Hoffman et al. 1969), gill lesions were mostly characterized by hyperplasia of the epithelium while skin lesions by a large number of dividing cells in the basal layer adjacent to the infected area. In our case, dividing cells could be occasionally seen near the infected area, though not to the extent described by Hoffman et al. (1969). The hypothesis of mechanical occlusion of respiration and osmoregulation in the gill interlamellar space has also been suggested in sea bass fingerlings in order to explain the mortalities experienced in an epitheliocystis-affected fish farm (Crespo et al. 2001).

The importance of epitheliocystis disease has greatly increased in the last years mainly due to the development of aquaculture and the introduction of new species. New molecular and immunological diagnostic techniques have also contributed significantly to the identification of the disease. Recently, epitheliocystis has begun to be considered a serious disease that could have a great impact on aquaculture production systems. This report enforces this argument, as it provides information about the effect of the disease in the early developmental stages for the first time. Furthermore, our observations strengthen the conclusion of Paperna (1977) that the disease is highly contagious in aquaculture systems, since all consecutively stocked mesocosm tanks of our facilities were eventually affected, with devastating results. Thus, proper hygiene and disinfection of the culture environment is highly advised.

\section{LITERATURE CITED}

> Athanassopoulou F, Prapas T, Rodger H (1999) Diseases of Puntazzo puntazzo Cuvier in marine aquaculture systems in Greece. J Fish Dis 22:215-218
Bennett HS, Wyrick AD, Lee SW, McNeil JH (1976) Science and art in preparing tissues embedded in plastic for light microscopy with special reference to glycol methacrylate, glass knives and simple stains. Stain Technol 51:71-94

Boglione C, Giganti M, Selmo C, Cataudella S (2003) Morphoecology in larval fin-fish: a new candidate species for aquaculture, Diplodus puntazzo (Sparidae). Aquacult Int 11:17-41

> Bradley TM, Newcomer CE, Maxwell KO (1988) Epitheliocystis associated with massive mortalities of cultured lake trout Salvelinus namaycush. Dis Aquat Org 4:9-17

> Crespo S, Grau A, Padrós F (1990) Epitheliocystis disease in the cultured amberjack, Seriola dumerili Risso (Carangidae). Aquaculture 90:197-207

> Crespo S, Zarza C, Padrós F, Marin De Mateo M (1999) Epitheliocystis agents in sea bream Sparus aurata: morphological evidence for two distinct chlamydia-like developmental cycles. Dis Aquat Org 37:61-72

Crespo S, Zarza C, Padrós F (2001) Epitheliocystis hyperinfection in sea bass, Dicentrarchus labrax (L.): light and electron microscope observations. J Fish Dis 24:557-560

Divanach P, Kentouri M, Charalambakis G, Pouget F, Sterioti A (1993) Comparison of growth performance of six Mediterranean fish species reared under intensive farming conditions in Crete (Greece), in raceways with the use of self feeders. In: Barnabé G, Kestemont P (eds) Production environment and quality. Bordeaux Aquaculture 1992. Spec Publ No. 18, European Aquaculture Society, Ghent, p 285-297

> Draghi A II, Bebak J, Popov VL, Noble AC and others (2007) Characterization of a Neochlamydia-like bacterium associated with epitheliocystis in cultured Arctic charr Salvelinus alpinus. Dis Aquat Org 76:27-38

Faranda F, Cavaliere A, Lo Paro G, Manganaro A, Mazzola A (1985) Preliminary studies on reproduction of Puntazzo puntazzo (Gmelin 1789) (Pisces, Sparidae) under controlled conditions. Aquaculture 49:111-123

Goodwin AE, Park E, Nowak BF (2005) Successful treatment of largemouth bass, Micropterus salmoides (L.), with epitheliocystis hyperinfection. J Fish Dis 28:623-625

Grau A, Crespo S (1991) Epitheliocystis in the wild and cultured amberjack, Seriola dumerili Risso: ultrastructural observations. Aquaculture 95:1-6

Hoffman GL, Dunbar CE, Wolf K, Zwillenberg LO (1969) Epitheliocystis, a new infectious disease of the bluegill (Lepomis macrochirus). Antonie Leeuwenhoek 35: 146-158

MacLean SA (1993) Pathological conditions of Narragansett Bay young-of-the-year winter flounder. Am Fish Soc Symp 14:47-54

McDowell EM, Trump BF (1976) Histologic fixatives suitable for diagnostic light and electron microscopy. Arch Pathol Lab Med 100:405-414

Munoz P, Cuesta A, Athanassopoulou F, Golomazou H and others (2007) Sharpsnout sea bream (Diplodus puntazzo) humoral immune response against the parasite Enteromyxum leei (Myxozoa). Fish Shellfish Immunol 23: 636-645

Nowak BF, Clark A (1999) Prevalence of epitheliocystis in Atlantic salmon, Salmo salar L., farmed in Tasmania, Australia. J Fish Dis 22:73-78

Nowak BF, LaPatra SE (2006) Epitheliocystis in fish. J Fish Dis 29:573-588

> Papandroulakis N, Kentouri M, Maingot E, Divanach P (2004) Mesocosm: a reliable technology for larval rearing of Diplodus puntazzo and Diplodus sargus sargus. Aquacult Int 12:345-355 
Paperna I (1977) Epitheliocystis infection in wild and cultured sea bream (Sparus aurata, Sparidae) and grey mullets (Liza ramada, Mugilidae). Aquaculture 10: 169-176

Sara M, Favaloro E, Mazzola A (1999) Comparative morphometrics of sharpsnout seabream (Diplodus puntazzo Cetti, 1777), reared in different conditions. Aquacult Eng 19: 195-209

Turnbull JF (1993) Epitheliocystis and salmonid rickettsial

Editorial responsibility: David Bruno,

Aberdeen, UK septicaemia. In: Inglis V, Roberts RJ, Bromage NR (eds) Bacterial diseases of fish. Blackwell Scientific, Oxford, p 237-254

> Varsamos S, Nebel C, Charmantier G (2005) Ontogeny of osmoregulation in postembryonic fish: a review. Comp Biochem Physiol A 141:401-429

Venizelos A, Benetti DD (1996) Epitheliocystis disease in cultured yellowtail Seriola mazatlana in Ecuador. J World Aquacult Soc 27:223-227

Submitted: May 21, 2008; Accepted: July 17, 2008

Proofs received from author(s): September 8, 2008 